\title{
VECTOR LATTICES ADMITTING A POSITIVELY HOMOGENEOUS CONTINUOUS FUNCTION CALCULUS
}

\author{
NIELS JAKOB LAUSTSEN AND VLADIMIR G. TROITSKY
}

\begin{abstract}
We characterize the Archimedean vector lattices that admit a positively homogeneous continuous function calculus by showing that the following two conditions are equivalent for each $n$-tuple $\boldsymbol{x}=\left(x_{1}, \ldots, x_{n}\right) \in X^{n}$, where $X$ is an Archimedean vector lattice and $n \in \mathbb{N}$ :
\end{abstract}

- there is a vector lattice homomorphism $\Phi_{x}: H_{n} \rightarrow X$ such that

$$
\Phi_{\boldsymbol{x}}\left(\pi_{i}^{(n)}\right)=x_{i} \quad(i \in\{1, \ldots, n\}),
$$

where $H_{n}$ denotes the vector lattice of positively homogeneous, continuous, realvalued functions defined on $\mathbb{R}^{n}$ and $\pi_{i}^{(n)}: \mathbb{R}^{n} \rightarrow \mathbb{R}$ is the $i^{\text {th }}$ coordinate projection;

- there is a positive element $e \in X$ such that $e \geqslant\left|x_{1}\right| \vee \cdots \vee\left|x_{n}\right|$ and the norm

$$
\|x\|_{e}=\inf \{\lambda \in[0, \infty):|x| \leqslant \lambda e\},
$$

defined for each $x$ in the order ideal $I_{e}$ of $X$ generated by $e$, is complete when restricted to the closed sublattice of $I_{e}$ generated by $x_{1}, \ldots, x_{n}$.

Moreover, we show that a vector space which admits a 'sufficiently strong' $H_{n}$-function calculus for each $n \in \mathbb{N}$ is automatically a vector lattice, and we explore the situation in the non-Archimedean case by showing that some non-Archimedean vector lattices admit a positively homogeneous continuous function calculus, while others do not.

\section{INTRODUCTION AND MAIN RESULTS}

Yudin [8] and Krivine [4] showed that every Banach lattice admits a positively homogeneous continuous function calculus. We refer to [5, Theorem 1.d.1] for an easily accessible, precise statement of this result, which is a fundamental tool in the study of Banach lattices, for instance allowing the definition of elements of the form $\left(\sum_{j=1}^{n}\left|x_{j}\right|^{p}\right)^{1 / p}$ for $p \in(1, \infty)$ whenever $n \in \mathbb{N}$ and $x_{1}, \ldots, x_{n}$ belong to some Banach lattice. Buskes, de Pagter and van Rooij [2, Theorem 3.7] have subsequently generalized the theorem of Yudin and Krivine to the class of uniformly complete Archimedean vector lattices.

2010 Mathematics Subject Classification. 06F20, 46A40 (primary); 46B42, 46E05 (secondary).

Key words and phrases. Vector lattice, positively homogeneous continuous function calculus, uniform completeness. 
After most of the research that this note is based upon was carried out, we learnt of a recent paper [3] of Buskes and Schwanke in which they study completions of Archimedean vector lattices with respect to a given non-empty collection $\mathcal{D}$ of positively homogeneous continuous functions. Of particular interest in the present context is their final result [3, Corollary 3.18], which states that for each such collection $\mathcal{D}$, every Archimedean vector lattice has a $\mathcal{D}$-completion, and it is unique up to vector lattice isomorphism.

The main aim of our work is to characterize the Archimedean vector lattices that admit a positively homogeneous continuous function calculus; we refer to Theorem 1.3 for a precise statement of this result. In combination with Proposition 3.1(i), it will in particular show that this class is strictly larger than that of uniformly complete Archimedean vector lattices. In the context of the work [3] of Buskes and Schwanke discussed above, Theorem 1.3 can be viewed as providing an alternative and perhaps more explicit description of the class of $\mathcal{D}$-complete Archimedean vector lattices in the special case where $\mathcal{D}$ is the collection of all positively homogeneous continuous functions.

In order to state our results precisely, we must introduce some notation and terminology. All vector spaces and lattices are assumed to be over the field $\mathbb{R}$ of real numbers. A real-valued function $f$ defined on a vector space $X$ is positively homogeneous if $f(\lambda x)=\lambda f(x)$ for each $\lambda \in[0, \infty)$ and each $x \in X$. For $n \in \mathbb{N}$, we denote by $H_{n}$ the vector lattice of positively homogeneous, continuous, real-valued functions defined on $\mathbb{R}^{n}$. The $i^{\text {th }}$ coordinate projection

$$
\pi_{i}^{(n)}:\left(t_{1}, \ldots, t_{n}\right) \mapsto t_{i}, \quad \mathbb{R}^{n} \rightarrow \mathbb{R},
$$

clearly belongs to $H_{n}$ for each $i \in\{1, \ldots, n\}$.

We can now clarify what we mean by 'a positively homogeneous continuous function calculus' for a general vector lattice.

Definition 1.1. A vector lattice $X$ admits a positively homogeneous continuous function calculus if, for each $n \in \mathbb{N}$ and each $n$-tuple $\boldsymbol{x}=\left(x_{1}, \ldots, x_{n}\right) \in X^{n}$, there is a vector lattice homomorphism $\Phi_{\boldsymbol{x}}: H_{n} \rightarrow X$ such that

$$
\Phi_{\boldsymbol{x}}\left(\pi_{i}^{(n)}\right)=x_{i} \quad(i \in\{1, \ldots, n\}) .
$$

In this case, we refer to the map $\boldsymbol{x} \mapsto \Phi_{\boldsymbol{x}}$ as a positively homogeneous continuous function calculus for $X$.

We shall next introduce a condition which turns out to be equivalent to admitting a positively homogeneous continuous function calculus. It involves the following standard 
notions. For a positive element $e$ of a vector lattice $X$, the set

$$
I_{e}=\{x \in X:|x| \leqslant \lambda e \text { for some } \lambda \in[0, \infty)\}
$$

is the order ideal generated by e, and

$$
\|x\|_{e}=\inf \{\lambda \in[0, \infty):|x| \leqslant \lambda e\} \quad\left(x \in I_{e}\right)
$$

defines a lattice seminorm on $I_{e}$. Suppose that $X$ is Archimedean, that is, whenever $x, y \in X^{+}$satisfy $n x \leqslant y$ for each $n \in \mathbb{N}$, it follows that $x=0$. Then $\|\cdot\|_{e}$ is a norm on $I_{e}$.

Definition 1.2. Let $X$ be an Archimedean vector lattice. Then:

- $X$ is uniformly complete if, for each positive element $e \in X$, the order ideal $I_{e}$ is complete with respect to the norm $\|\cdot\|_{e}$ given by (1.3);

- $X$ is finitely uniformly complete if, for each $n \in \mathbb{N}$ and $x_{1}, \ldots, x_{n} \in X$, there is a positive element $e \in X$ such that $e \geqslant\left|x_{1}\right| \vee \cdots \vee\left|x_{n}\right|$ and the norm $\|\cdot\|_{e}$ is complete on the closed sublattice of $\left(I_{e},\|\cdot\|_{e}\right)$ generated by $x_{1}, \ldots, x_{n}$.

The first of these two notions is standard, whereas the second appears to be new. Clearly, the first implies the second; Proposition 3.1(i) below will show that they are not equivalent.

We are now ready to state our two main results.

Theorem 1.3. Let $X$ be an Archimedean vector lattice. Then $X$ admits a positively homogeneous continuous function calculus if and only if $X$ is finitely uniformly complete.

When $X$ is finitely uniformly complete, the positively homogeneous continuous function calculus is unique (in the sense that for each $n \in \mathbb{N}$ and $\boldsymbol{x} \in X^{n}$, there is only one vector lattice homomorphism $\Phi_{\boldsymbol{x}}: H_{n} \rightarrow X$ which satisfies (1.1)), and

$$
\Phi_{\left(\Phi_{\boldsymbol{x}}\left(f_{1}\right), \ldots, \Phi_{\boldsymbol{x}}\left(f_{m}\right)\right)}(g)=\Phi_{\boldsymbol{x}}\left(g \circ\left(f_{1} \times \cdots \times f_{m}\right)\right)
$$

for each $m, n \in \mathbb{N}, \boldsymbol{x} \in X^{n}, f_{1}, \ldots, f_{m} \in H_{n}$ and $g \in H_{m}$, where $f_{1} \times \cdots \times f_{m}: \mathbb{R}^{n} \rightarrow \mathbb{R}^{m}$ is the function defined by

$$
\left(f_{1} \times \cdots \times f_{m}\right)(t)=\left(f_{1}(t), \ldots, f_{m}(t)\right) \quad\left(t \in \mathbb{R}^{n}\right) .
$$

Writing $f(\boldsymbol{x})$ for $\Phi_{\boldsymbol{x}}(f),(1.4)$ takes the more suggestive form

$$
g\left(f_{1}(\boldsymbol{x}), \ldots, f_{m}(\boldsymbol{x})\right)=\left(g \circ\left(f_{1} \times \cdots \times f_{m}\right)\right)(\boldsymbol{x}) .
$$

Our other main result states that a vector space which admits a 'sufficiently strong' function calculus is automatically a vector lattice with a positively homogeneous continuous function calculus. 
Theorem 1.4. Let $X$ be a vector space and suppose that, for each $n \in \mathbb{N}$ and each $n$-tuple $\boldsymbol{x} \in X^{n}$, there is a linear map $\Phi_{\boldsymbol{x}}: H_{n} \rightarrow X$ which satisfies conditions (1.1) and (1.4). Then $X$ admits the structure of a vector lattice, and $\boldsymbol{x} \mapsto \Phi_{\boldsymbol{x}}$ is a positively homogeneous continuous function calculus for $X$ with respect to this lattice structure. Moreover, $X$ is Archimedean if and only if $\operatorname{ker} \Phi_{\boldsymbol{x}}$ is closed in $H_{n}$ for each $n \in \mathbb{N}$ and $\boldsymbol{x} \in X^{n}$, where $H_{n}$ is given the topology obtained by identifying it with $C\left(S_{\ell_{\infty}^{n}}\right)$ (see (2.2) for details of this identification).

The notion of (finite) uniform completeness does not extend easily to the non-Archimedean setting. Indeed, if $x, y \in X^{+} \backslash\{0\}$ satisfy $n x \leqslant y$ for each $n \in \mathbb{N}$, then for any $e \in X^{+}$such that $e \geqslant x \vee y$, we have $\|x\|_{e}=0$ because $n x \leqslant y \leqslant e$ for each $n \in \mathbb{N}$. Hence $\|\cdot\|_{e}$ is only a seminorm (even when it is restricted to the sublattice generated by $x$ and $y$ ).

This raises the question whether a non-Archimedean vector lattice may admit a positively homogeneous continuous function calculus. We shall address it in Section 3, where on the one hand Proposition 3.1(ii) will show that certain non-Archimedean vector lattices do admit a positively homogeneous continuous function calculus which satisfies (1.4), while on the other Example 3.4 will exhibit a non-Archimedean vector lattice that does not admit any positively homogeneous continuous function calculus. We observe that the former of these two results implies that the condition in the final clause of Theorem 1.4 is not always satisfied.

\section{Proofs of Theorems 1.3 And 1.4}

For a topological space $K$, we write $C(K)$ for the vector lattice of continuous, real-valued functions defined on $K$. This is of course a Banach lattice with respect to the supremum norm $\|\cdot\|_{\infty}$ when $K$ is a compact Hausdorff space. We use the symbol $\mathbf{1}$ to denote the constant function 1 defined on $K$.

Lemma 2.1. Let $T: C(K) \rightarrow X$ be a vector lattice homomorphism, where $K$ is a compact Hausdorff space and $X$ is an Archimedean vector lattice, and set $e=T(\mathbf{1}) \in X^{+}$. Then $T[C(K)] \subseteq I_{e}, T$ is continuous with operator norm at most 1 when regarded as a map into $\left(I_{e},\|\cdot\|_{e}\right)$, and the restriction of the norm $\|\cdot\|_{e}$ to $T[(C(K)]$ is complete.

Proof. Each $f \in C(K)$ satisfies $|f| \leqslant\|f\|_{\infty} \mathbf{1}$, so as $T$ is a vector lattice homomorphism, we have

$$
\|f\|_{\infty} e=T\left(\|f\|_{\infty} \mathbf{1}\right) \geqslant T(|f|)=|T(f)| .
$$

This shows that $T(f) \in I_{e}$ with $\|T(f)\|_{e} \leqslant\|f\|_{\infty}$, so that $T[C(K)] \subseteq I_{e}$ and $T$ is continuous with operator norm at most 1 when regarded as a map into $\left(I_{e},\|\cdot\|_{e}\right)$. 
We shall verify that the restriction of the norm $\|\cdot\|_{e}$ to $T[(C(K)]$ is complete by showing that each absolutely convergent series $\sum_{n=1}^{\infty} x_{n}$ in $\left(T\left[(C(K)],\|\cdot\|_{e}\right)\right.$ converges. It suffices to consider the case where $x_{n}$ is positive for each $n \in \mathbb{N}$ because $\|\cdot\|_{e}$ is a lattice norm. Then we can take $f_{n} \in C(K)^{+}$such that $T f_{n}=x_{n}$. Set $g_{n}=f_{n} \wedge\left\|x_{n}\right\|_{e} \mathbf{1}$ and observe that $\left\|g_{n}\right\|_{\infty} \leqslant\left\|x_{n}\right\|_{e}$, so that the series $\sum_{n=1}^{\infty} g_{n}$ is absolutely convergent and therefore convergent in $C(K)$; denote its sum by $g$. We see that $T g_{n}=x_{n} \wedge\left\|x_{n}\right\|_{e} e=x_{n}$ because $x_{n} \leqslant\left\|x_{n}\right\|_{e} e$. Since $T$ is continuous and linear, we conclude that the series $\sum_{n=1}^{\infty} x_{n}$ converges to $\mathrm{Tg}$ in $\left(T[C(K)],\|\cdot\|_{e}\right)$, and the result follows.

For $n \in \mathbb{N}$, the unit sphere

$$
S_{\ell_{\infty}^{n}}=\left\{\left(t_{1}, \ldots, t_{n}\right) \in \mathbb{R}^{n}: \max _{1 \leqslant j \leqslant n}\left|t_{j}\right|=1\right\}
$$

of the Banach space $\ell_{\infty}^{n}$ is a compact metric space with respect to the metric $d$ induced by the norm, that is,

$$
d\left(\left(s_{1}, \ldots, s_{n}\right),\left(t_{1}, \ldots, t_{n}\right)\right)=\max _{1 \leqslant j \leqslant n}\left|s_{j}-t_{j}\right| .
$$

It is well known and easy to see that the restriction map

$$
f \mapsto f\left\lceil_{\ell_{\infty}^{n}}\right.
$$

is a vector lattice isomorphism of $H_{n}$ onto $C\left(S_{\ell_{\infty}^{n}}\right)$, where we recall that $H_{n}$ denotes the sublattice of $C\left(\mathbb{R}^{n}\right)$ of positively homogeneous functions. Hence we may identify $H_{n}$ with the Banach lattice $C\left(S_{\ell_{\infty}^{n}}\right)$. Although we do not require this result, we remark that de Pagter and Wickstead [6, Proposition 5.3] have shown that this Banach lattice is isomorphic to the free Banach lattice on $n$ generators.

The following result can be viewed as a generalization of [2, Theorem 3.7].

Proposition 2.2. Let $X$ be an Archimedean vector lattice, and let $\boldsymbol{x}=\left(x_{1}, \ldots, x_{n}\right) \in X^{n}$ for some $n \in \mathbb{N}$. Then the following three conditions are equivalent:

(a) there is a vector lattice homomorphism $\Phi_{\boldsymbol{x}}: H_{n} \rightarrow X$ which satisfies (1.1);

(b) the norm $\|\cdot\|_{e}$ is complete on the closed sublattice of $\left(I_{e},\|\cdot\|_{e}\right)$ generated by the elements $x_{1}, \ldots, x_{n}$, where $e=\left|x_{1}\right| \vee \cdots \vee\left|x_{n}\right|$;

(c) there is a positive element $e \in X$ such that $e \geqslant\left|x_{1}\right| \vee \cdots \vee\left|x_{n}\right|$ and the norm $\|\cdot\|_{e}$ is complete on the closed sublattice of $\left(I_{e},\|\cdot\|_{e}\right)$ generated by $x_{1}, \ldots, x_{n}$.

When one and hence all three of these conditions are satisfied, the vector lattice homomorphism $\Phi_{\boldsymbol{x}}: H_{n} \rightarrow X$ satisfying (1.1) is unique.

Proof. Throughout the proof we shall freely identify $H_{n}$ with $C\left(S_{\ell_{\infty}^{n}}\right)$ via the map (2.2). 
$(\mathrm{a}) \Rightarrow(\mathrm{b})$. Suppose that $\Phi_{x}: C\left(S_{\ell_{\infty}^{n}}\right) \rightarrow X$ is a vector lattice homomorphism which satisfies (1.1). Then, applying $\Phi_{\boldsymbol{x}}$ to the identity

$$
\left.\left|\pi_{1}^{(n)} \uparrow_{S_{\ell_{\infty}^{n}} \mid \vee \cdots \vee}\right| \pi_{n}^{(n)}\right|_{S_{\ell_{\infty}^{n}}} \mid=\mathbf{1}
$$

we obtain

$$
\Phi_{\boldsymbol{x}}(\mathbf{1})=\left|\Phi_{\boldsymbol{x}}\left(\pi_{1}^{(n)} \uparrow_{S_{\ell_{\infty}^{n}}}\right)\right| \vee \cdots \vee\left|\Phi_{\boldsymbol{x}}\left(\pi_{n}^{(n)} \uparrow_{S_{\ell_{\infty}^{n}}}\right)\right|=\left|x_{1}\right| \vee \cdots \vee\left|x_{n}\right|=e .
$$

Hence Lemma 2.1 implies that $\Phi_{x}\left[C\left(S_{\ell_{\infty}^{n}}\right)\right] \subseteq I_{e}$ and the restriction of the norm $\|\cdot\|_{e}$ to $\Phi_{\boldsymbol{x}}\left[C\left(S_{\ell_{\infty}^{n}}\right)\right]$ is complete, and therefore (b) is satisfied because $x_{1}, \ldots, x_{n} \in \Phi_{\boldsymbol{x}}\left[C\left(S_{\ell_{\infty}^{n}}\right)\right]$.

$(\mathrm{b}) \Rightarrow(\mathrm{c})$. This is clear.

$(\mathrm{c}) \Rightarrow(\mathrm{a})$. Suppose that $e \in X^{+}$satisfies $e \geqslant\left|x_{1}\right| \vee \cdots \vee\left|x_{n}\right|$ and the norm $\|\cdot\|_{e}$ is complete on the closed sublattice $X_{\boldsymbol{x}}$ of $I_{e}$ generated by $x_{1}, \ldots, x_{n}$. Then $\left(X_{\boldsymbol{x}},\|\cdot\|_{e}\right)$ is a Banach lattice containing $x_{1}, \ldots, x_{n}$, so the Yudin/Krivine Theorem as it is stated in [5, Theorem 1.d.1] implies that there is a vector lattice homomorphism $\Phi_{\boldsymbol{x}}: H_{n} \rightarrow X_{\boldsymbol{x}}$ which satisfies (1.1). This remains true if we consider $\Phi_{\boldsymbol{x}}$ as a map into the larger codomain $X$, so that (a) is satisfied.

To prove the final clause, suppose that (a)-(c) hold, and let $\Phi_{\boldsymbol{x}}: C\left(S_{\ell_{\infty}^{n}}\right) \rightarrow X$ be a vector lattice homomorphism which satisfies (1.1). Then $\Phi_{\boldsymbol{x}}$ is uniquely determined on the sublattice of $C\left(S_{\ell_{\infty}^{n}}\right)$ generated by $\pi_{1}^{(n)} \uparrow_{S_{\ell_{\infty}^{n}}}, \ldots, \pi_{n}^{(n)} \uparrow_{S_{\ell_{\infty}^{n}}}$. The Stone-Weierstrass Theorem implies that this sublattice is dense in $C\left(S_{\ell_{\infty}^{n}}\right)$ because it separates the points of $S_{\ell_{\infty}^{n}}$ and contains 1 by (2.3). As in the proof of $(\mathrm{a}) \Rightarrow(\mathrm{b})$ above, we see that Lemma 2.1 applies; it shows that $\Phi_{\boldsymbol{x}}$ is continuous when regarded as an operator into $\left(I_{e},\|\cdot\|_{e}\right)$, and therefore $\Phi_{\boldsymbol{x}}$ is uniquely determined on all of $C\left(S_{\ell_{\infty}^{n}}\right)$.

Proof of Theorem 1.3. The equivalence of conditions (a) and (c) in Proposition 2.2 implies immediately that $X$ admits a positively homogeneous continuous function calculus if and only if it is finitely uniformly complete.

To prove (1.4), we begin by remarking that for $m, n \in \mathbb{N}$ and $f_{1}, \ldots, f_{m} \in H_{n}$, the function $f_{1} \times \cdots \times f_{m}$ given by (1.5) is continuous and the composition $g \circ\left(f_{1} \times \cdots \times f_{m}\right)$ is positively homogeneous for each $g \in H_{m}$. Hence, for $\boldsymbol{x} \in X^{n}$, we have a map

$$
g \mapsto \Phi_{x}\left(g \circ\left(f_{1} \times \cdots \times f_{m}\right)\right), \quad H_{m} \rightarrow X .
$$

This map is a vector lattice homomorphism which maps $\pi_{i}^{(m)}$ to $\Phi_{\boldsymbol{x}}\left(f_{i}\right)$ for each $i \in$ $\{1, \ldots, m\}$ because $\pi_{i}^{(m)} \circ\left(f_{1} \times \cdots \times f_{m}\right)=f_{i}$, and it is therefore equal to $\Phi_{\left(\Phi_{\boldsymbol{x}}\left(f_{1}\right), \ldots, \Phi_{\boldsymbol{x}}\left(f_{m}\right)\right)}$ by the uniqueness statement in the last clause of Proposition 2.2.

We shall next prove Theorem 1.4. This will involve the following easy and undoubtedly well-known lemma. 
Lemma 2.3. Let $Y$ be a sublattice of a normed vector lattice $X$, and suppose that

$$
\bar{Y} \cap X^{+} \subseteq Y
$$

Then $Y$ is closed.

Proof. Let $y \in \bar{Y}$. Since $\bar{Y}$ is a sublattice, $y^{ \pm} \in \bar{Y}$, and they are both positive by their definitions. Hence $y^{ \pm} \in Y$ by the assumption, and therefore $y=y^{+}-y^{-} \in Y$.

Proof of Theorem 1.4. Throughout this proof, we shall write $f(\boldsymbol{x})$ instead of $\Phi_{\boldsymbol{x}}(f)$. In this notation, the linearity of $\Phi_{\boldsymbol{x}}$ translates into the statement

$$
(f+\lambda g)(\boldsymbol{x})=f(\boldsymbol{x})+\lambda g(\boldsymbol{x}) \quad\left(n \in \mathbb{N}, \boldsymbol{x} \in X^{n}, f, g \in H_{n}, \lambda \in \mathbb{R}\right),
$$

while (1.1) becomes

$$
\pi_{i}^{(n)}\left(x_{1}, \ldots, x_{n}\right)=x_{i} \quad\left(n \in \mathbb{N}, i \in\{1, \ldots, n\}, x_{1}, \ldots, x_{n} \in X\right),
$$

and (1.6) replaces (1.4).

The map $\sigma:\left(t_{1}, t_{2}\right) \mapsto t_{1} \vee t_{2}, \mathbb{R}^{2} \rightarrow \mathbb{R}$, belongs to $H_{2}$, so that $\sigma\left(x_{1}, x_{2}\right)$ defines an element of $X$ for each pair $x_{1}, x_{2} \in X$. Consequently, we may define a relation $\leqslant$ on $X$ by

$$
x_{1} \leqslant x_{2} \Longleftrightarrow \sigma\left(x_{1}, x_{2}\right)=x_{2} \quad\left(x_{1}, x_{2} \in X\right) .
$$

Our first aim is to show that this relation is a partial order on $X$.

Reflexivity. The fact that $\sigma(t, t)=t$ for every $t \in \mathbb{R}$ implies that $\sigma \circ\left(\pi_{1}^{(2)} \times \pi_{1}^{(2)}\right)=\pi_{1}^{(2)}$ in $H_{2}$. Hence, by (1.6) and (2.5), we obtain

$$
\sigma(x, x)=x \quad(x \in X),
$$

which shows that $x \leqslant x$ for each $x \in X$, as required.

AnTI-SYMMETRY. Since $\sigma\left(t_{1}, t_{2}\right)=\sigma\left(t_{2}, t_{1}\right)$ for every pair $t_{1}, t_{2} \in \mathbb{R}$, we have $\sigma=$ $\sigma \circ\left(\pi_{2}^{(2)} \times \pi_{1}^{(2)}\right)$ in $H_{2}$. Combining this identity with (1.6) and (2.5), we see that

$$
\sigma\left(x_{1}, x_{2}\right)=\sigma\left(\pi_{2}^{(2)}\left(x_{1}, x_{2}\right), \pi_{1}^{(2)}\left(x_{1}, x_{2}\right)\right)=\sigma\left(x_{2}, x_{1}\right) \quad\left(x_{1}, x_{2} \in X\right) .
$$

Now suppose that $x_{1}, x_{2} \in X$ satisfy $x_{1} \leqslant x_{2}$ and $x_{2} \leqslant x_{1}$. Then $\sigma\left(x_{1}, x_{2}\right)=x_{2}$ and $\sigma\left(x_{2}, x_{1}\right)=x_{1}$, so that $x_{1}=x_{2}$ by $(2.7)$, as required.

TRANSITIVITY. The associativity of $\vee$ means that $\sigma\left(t_{1}, \sigma\left(t_{2}, t_{3}\right)\right)=\sigma\left(\sigma\left(t_{1}, t_{2}\right), t_{3}\right)$ for every $t_{1}, t_{2}, t_{3} \in \mathbb{R}$. Hence the identity

$$
\sigma \circ\left(\pi_{1}^{(3)} \times\left(\sigma \circ\left(\pi_{2}^{(3)} \times \pi_{3}^{(3)}\right)\right)\right)=\sigma \circ\left(\left(\sigma \circ\left(\pi_{1}^{(3)} \times \pi_{2}^{(3)}\right)\right) \times \pi_{3}^{(3)}\right)
$$

holds in $\mathrm{H}_{3}$, from which we deduce that

$$
\sigma\left(x_{1}, \sigma\left(x_{2}, x_{3}\right)\right)=\sigma\left(\sigma\left(x_{1}, x_{2}\right), x_{3}\right) \quad\left(x_{1}, x_{2}, x_{3} \in X\right)
$$


by (1.6) and (2.5). Now suppose that $x_{1}, x_{2}, x_{3} \in X$ satisfy $x_{1} \leqslant x_{2}$ and $x_{2} \leqslant x_{3}$. Then we have $\sigma\left(x_{1}, x_{2}\right)=x_{2}$ and $\sigma\left(x_{2}, x_{3}\right)=x_{3}$. Substituting these identities into (2.8), we obtain $\sigma\left(x_{1}, x_{3}\right)=\sigma\left(x_{2}, x_{3}\right)=x_{3}$, which shows that $x_{1} \leqslant x_{3}$, as required.

Having thus established that $\leqslant$ is a partial order, we shall next show that each pair $\left(x_{1}, x_{2}\right)$ of elements of $X$ has a supremum with respect to $\leqslant$, and it is given by $\sigma\left(x_{1}, x_{2}\right)$. To this end, we observe that (2.8) and (2.6) imply that

$$
\sigma\left(x_{1}, \sigma\left(x_{1}, x_{2}\right)\right)=\sigma\left(\sigma\left(x_{1}, x_{1}\right), x_{2}\right)=\sigma\left(x_{1}, x_{2}\right),
$$

so that $x_{1} \leqslant \sigma\left(x_{1}, x_{2}\right)$. A similar argument, using also (2.7), shows that $x_{2} \leqslant \sigma\left(x_{1}, x_{2}\right)$, and therefore $\sigma\left(x_{1}, x_{2}\right)$ is an upper bound of the pair $\left(x_{1}, x_{2}\right)$. To show that it is the least upper bound, suppose that $y \in X$ satisfies $x_{1} \leqslant y$ and $x_{2} \leqslant y$, so that $\sigma\left(x_{1}, y\right)=y=\sigma\left(x_{2}, y\right)$. Then we have $\sigma\left(x_{1}, x_{2}\right) \leqslant y$ because (2.8) implies that

$$
\sigma\left(\sigma\left(x_{1}, x_{2}\right), y\right)=\sigma\left(x_{1}, \sigma\left(x_{2}, y\right)\right)=\sigma\left(x_{1}, y\right)=y
$$

as required.

It remains to verify that $\leqslant$ is positively homogeneous and translation-invariant.

Positive homogeneity. Suppose that $x_{1}, x_{2} \in X$ satisfy $x_{1} \leqslant x_{2}$, and let $\lambda \in[0, \infty)$. The positive homogeneity of $\sigma$ translates into the identity $\lambda \sigma=\sigma \circ\left(\lambda \pi_{1}^{(2)} \times \lambda \pi_{2}^{(2)}\right)$ in $H_{2}$, and therefore we have

$$
\sigma\left(\lambda x_{1}, \lambda x_{2}\right)=(\lambda \sigma)\left(x_{1}, x_{2}\right)=\lambda \sigma\left(x_{1}, x_{2}\right)=\lambda x_{2}
$$

by (1.6), (2.4) and (2.5). This shows that $\lambda x_{1} \leqslant \lambda x_{2}$.

Translation Invariance. Since $\sigma\left(t_{1}+t_{3}, t_{2}+t_{3}\right)=\sigma\left(t_{1}, t_{2}\right)+t_{3}$ for every $t_{1}, t_{2}, t_{3} \in \mathbb{R}$, we have

$$
\sigma \circ\left(\left(\pi_{1}^{(3)}+\pi_{3}^{(3)}\right) \times\left(\pi_{2}^{(3)}+\pi_{3}^{(3)}\right)\right)=\sigma \circ\left(\pi_{1}^{(3)} \times \pi_{2}^{(3)}\right)+\pi_{3}^{(3)}
$$

in $H_{3}$. Let $x_{1}, x_{2}, x_{3} \in X$, suppose that $x_{1} \leqslant x_{2}$, and set $\boldsymbol{x}=\left(x_{1}, x_{2}, x_{3}\right)$. Then we have

$$
\begin{aligned}
\sigma\left(x_{1}+x_{3}, x_{2}+x_{3}\right) & =\left(\sigma \circ\left(\pi_{1}^{(3)} \times \pi_{2}^{(3)}\right)+\pi_{3}^{(3)}\right)(\boldsymbol{x}) & & \text { by }(1.6),(2.4),(2.5) \text { and }(2.9) \\
& =\sigma\left(x_{1}, x_{2}\right)+x_{3} & & \text { by }(1.6),(2.4) \text { and }(2.5) \\
& =x_{2}+x_{3} & & \text { because } x_{1} \leqslant x_{2}
\end{aligned}
$$

so that $x_{1}+x_{3} \leqslant x_{2}+x_{3}$, as required.

This completes the proof that $X$ is a vector lattice with respect to the order $\leqslant$.

To show that $\Phi_{\boldsymbol{x}}$ is a vector lattice homomorphism for every $n \in \mathbb{N}$ and $\boldsymbol{x} \in X^{n}$, take $f, g \in H_{n}$. Then $f \vee g=\sigma \circ(f \times g)$ in $H_{n}$, so that $(f \vee g)(\boldsymbol{x})=\sigma(f(\boldsymbol{x}), g(\boldsymbol{x}))$ by (1.6). Recalling the convention that $\Phi_{\boldsymbol{x}}(h)=h(\boldsymbol{x})$ for $h \in H_{n}$ and that the supremum in $X$ is 
given by $x_{1} \vee x_{2}=\sigma\left(x_{1}, x_{2}\right)$ for $x_{1}, x_{2} \in X$, we see that this means that $\Phi_{\boldsymbol{x}}$ preserves suprema. Hence the conclusion follows because $\Phi_{\boldsymbol{x}}$ is linear by assumption.

We shall prove the forward implication $(\Rightarrow)$ of the final statement by contraposition. Suppose that $\operatorname{ker} \Phi_{\boldsymbol{x}}$ is not closed in $H_{n}$ for some $n \in \mathbb{N}$ and $\boldsymbol{x} \in X^{n}$. By Lemma 2.3, we can choose $f \in H_{n}^{+} \cap \overline{\operatorname{ker} \Phi_{\boldsymbol{x}}} \backslash \operatorname{ker} \Phi_{\boldsymbol{x}}$, which implies that $\Phi_{\boldsymbol{x}}(f) \in X^{+} \backslash\{0\}$. For each $m \in \mathbb{N}$, take $f_{m} \in \operatorname{ker} \Phi_{\boldsymbol{x}}$ such that $\left\|f-f_{m}\right\|_{\infty} \leqslant 1 / m$. Then we have $m\left(f-f_{m}\right) \leqslant \mathbf{1}$, so that

$$
m \Phi_{\boldsymbol{x}}(f)=\Phi_{\boldsymbol{x}}\left(m\left(f-f_{m}\right)\right) \leqslant \Phi_{\boldsymbol{x}}(\mathbf{1}) \quad(m \in \mathbb{N}) .
$$

This shows that $X$ is not Archimedean.

Conversely, suppose that $\operatorname{ker} \Phi_{\boldsymbol{x}}$ is closed in $H_{n}$ for each $n \in \mathbb{N}$ and $\boldsymbol{x} \in X^{n}$. Further, suppose that $x_{1}, x_{2} \in X^{+}$satisfy $m x_{1} \leqslant x_{2}$ for each $m \in \mathbb{N}$, and set $\boldsymbol{x}=\left(x_{1}, x_{2}\right) \in X^{2}$. The Fundamental Isomorphism Theorem implies that the vector lattices $\Phi_{\boldsymbol{x}}\left[H_{2}\right]$ and $H_{2} / \operatorname{ker} \Phi_{\boldsymbol{x}}$ are isomorphic. Since the order ideal $\operatorname{ker} \Phi_{\boldsymbol{x}}$ is closed by the assumption, the quotient $H_{2} / \operatorname{ker} \Phi_{\boldsymbol{x}}$ is a Banach lattice and thus Archimedean. Therefore $\Phi_{\boldsymbol{x}}\left[H_{2}\right]$ is also Archimedean, from which we conclude that $x_{1}=0$ because $x_{1}, x_{2} \in \Phi_{x}\left[H_{2}\right]$.

\section{EXAMPLES}

The purpose of this section is to present three examples that complement Theorems 1.3 and 1.4. The first of these examples shows that there are finitely uniformly complete Archimedean vector lattices which are not uniformly complete, while the second and third

explore the situation for non-Archimedean vector lattices by demonstrating that some non-Archimedean vector lattices admit a positively homogeneous continuous function calculus satisfying (1.4), whereas others do not admit any positively homogeneous continuous function calculus.

Since the first two of these examples work in the generality of an arbitrary infinite compact Hausdorff space, we have chosen to formally label them 'proposition' instead of 'example', even though they serve as illustrative examples in the context of this paper. Moreover, as they share a common framework and have several parts of their proofs in common, we shall state them as a single proposition rather than two.

Proposition 3.1. Let $K$ be a compact Hausdorff space of infinite cardinality, and choose an accumulation point $s_{0} \in K$ of a countably infinite subset of $K$.

(i) The set

$$
X=\left\{f \in C(K): f \uparrow_{U} \text { is constant for some neighbourhood } U \text { of } s_{0}\right\}
$$


is a proper, dense sublattice of $C(K)$ and thus Archimedean, and $X$ is finitely uniformly complete, but not uniformly complete.

(ii) The set

$$
J=\left\{f \in C(K): f \uparrow_{U}=0 \text { for some neighbourhood } U \text { of } s_{0}\right\}
$$

is an order ideal of $C(K)$; it is not closed, and the quotient $C(K) / J$ is a non-Archimedean vector lattice which admits a positively homogeneous function calculus that satisfies (1.4).

Proof. Being infinite, $K$ contains a countably infinite subset $S$, and the compactness of $K$ implies that $S$ has an accumulation point $s_{0} \in K$.

(i). It is straightforward to verify that $X$ is a sublattice of $C(K)$.

To show that $X \neq C(K)$, we begin by enumerating $S \backslash\left\{s_{0}\right\}$ as $\left\{s_{m}: m \in \mathbb{N}\right\}$, where $s_{m} \neq s_{n}$ for $m \neq n$. Then, for each $m \in \mathbb{N}$, Urysohn's Lemma implies that there is a continuous function $h_{m}: K \rightarrow[0,1]$ with $h_{m}\left(s_{0}\right)=0$ and $h_{m}\left(s_{m}\right)=1$. Define

$$
h=\sum_{m=1}^{\infty} 2^{-m} h_{m} \in C(K)^{+} .
$$

For each neighbourhood $U$ of $s_{0}$, we can choose $m \in \mathbb{N}$ such that $s_{m} \in U$, and we have

$$
h\left(s_{m}\right) \geqslant \frac{1}{2^{m}}>0=h\left(s_{0}\right) .
$$

This shows that $h \uparrow_{U}$ is not constant, so that $h \notin X$.

Next, to establish the density of $X$ in $C(K)$, we shall prove that for each $\varepsilon>0$ and $f \in C(K)$, we can find a neighbourhood $U$ of $s_{0}$ and a function $g \in C(K)$ such that

$$
\|f-g\|_{\infty} \leqslant \varepsilon \quad \text { and } \quad g(u)=f\left(s_{0}\right) \quad(u \in U) .
$$

Indeed, since $f$ is continuous at $s_{0}$, we can choose an open neighbourhood $V$ of $s_{0}$ such that $\left|f(v)-f\left(s_{0}\right)\right| \leqslant \varepsilon$ for each $v \in V$. Being compact, $K$ is regular, so there is an open neighbourhoood $U$ of $s_{0}$ with $\bar{U} \subseteq V$. Urysohn's Lemma then produces a continuous function $k: K \rightarrow[0,1]$ such that $k(u)=1$ for each $u \in \bar{U}$ and $k(t)=0$ for each $t \in K \backslash V$. Set $g=f\left(s_{0}\right) k+(1-k) f \in C(K)$. Clearly $g(u)=f\left(s_{0}\right)$ for each $u \in \bar{U}$, and

$$
|f(v)-g(v)|=\left|f(v)-f\left(s_{0}\right)\right||k(v)| \leqslant \varepsilon \quad(v \in V) \quad \text { and } \quad g \uparrow_{K \backslash V}=f \uparrow_{K \backslash V},
$$

which implies that (3.4) is satisfied.

Since $X$ contains the constant functions, the order ideal $I_{1}$ of $X$ generated by the constant function 1 is equal to $X$, and the associated norm $\|\cdot\|_{1}$ given by (1.3) is equal to the supremum norm $\|\cdot\|_{\infty}$. This implies that $\left(I_{\mathbf{1}},\|\cdot\|_{1}\right)$ is not complete because $X$ is a proper, dense subset of $C(K)$, and therefore $X$ is not uniformly complete. 
To verify that $X$ is finitely uniformly complete, let $f_{1}, \ldots, f_{n} \in X$ for some $n \in \mathbb{N}$, and take a neighbourhood $U$ of $s_{0}$ such that the restrictions $f_{1} \uparrow_{U}, \ldots, f_{n} \uparrow_{U}$ are all constant. We may suppose that $f_{1}, \ldots, f_{n}$ are not all 0 , so that $c:=\max _{1 \leqslant j \leqslant n}\left\|f_{j}\right\|_{\infty}>0$. Then $e:=c \mathbf{1}$ satisfies $e \geqslant\left|f_{1}\right| \vee \cdots \vee\left|f_{n}\right|$ and $\|g\|_{e}=c^{-1}\|g\|_{\infty}$ for each $g \in X$. Moreover, the set

$$
Y=\left\{f \in C(K): f \uparrow_{U} \text { is constant }\right\}
$$

is a closed sublattice of $C(K)$ such that $e, f_{1}, \ldots, f_{n} \in Y \subseteq X$. In particular, the norm $\|\cdot\|_{e}$ is complete on $Y$ and therefore also on the closed sublattice generated by $f_{1}, \ldots, f_{n}$. This proves that $X$ is finitely uniformly complete.

(ii). It is easily checked that $J$ is an order ideal of $C(K)$. We claim that its closure is given by

$$
\bar{J}=\left\{f \in C(K): f\left(s_{0}\right)=0\right\} .
$$

Indeed, the set on the right-hand side is clearly a closed set containing $J$, and (3.4) implies that each $f \in C(K)$ with $f\left(s_{0}\right)=0$ can be approximated arbitrarily well by elements of $J$. This proves (3.5), from which we deduce that $J$ is not closed because (3.3) shows that the function $h$ given by (3.2) belongs to $\bar{J} \backslash J$.

Set $Z=C(K) / J$, and let $Q: C(K) \rightarrow Z$ be the quotient homomorphism. To prove that $Z$ is not Archimedean, set $g_{n}=\left(h-\frac{1}{n} \mathbf{1}\right)^{+} \in C(K)^{+}$for each $n \in \mathbb{N}$, where $h$ is defined by (3.2), as above. We see that $g_{n} \in J$ because $h$ is continuous with $h\left(s_{0}\right)=0$. Hence the inequality $\mathbf{1} \geqslant n\left(h-g_{n}\right)$ implies that $Q(\mathbf{1}) \geqslant n Q(h)$ for each $n \in \mathbb{N}$. However, $Q(h) \neq 0$ because $h \notin J$, and therefore $Z$ is not Archimedean.

Being a Banach lattice, $C(K)$ has a positively homogeneous continuous function calculus which satisfies (1.4). More precisely, in the particular case of a $C(K)$-space, it is easy to see that the positively homogeneous continuous function calculus takes the following explicit form:

$$
\left(\Phi_{\boldsymbol{f}}(g)\right)(t)=g\left(f_{1}(t), \ldots, f_{n}(t)\right) \quad(t \in K)
$$

for $n \in \mathbb{N}, \boldsymbol{f}=\left(f_{1}, \ldots, f_{n}\right) \in C(K)^{n}$ and $g \in H_{n}$.

Let $R: Z \rightarrow C(K)$ be a right inverse map of $Q$. (We can choose $R$ to be linear if we wish, but not in general a vector lattice homomorphism, of course.) Then, for each $n \in \mathbb{N}$ and $\boldsymbol{z}=\left(z_{1}, \ldots, z_{n}\right) \in Z^{n}$, we can define a vector lattice homomorphism by

$$
\Phi_{\boldsymbol{z}}=Q \circ \Phi_{\left(R z_{1}, \ldots, R z_{n}\right)}: H_{n} \rightarrow Z
$$

It satisfies (1.1) because

$$
\Phi_{\boldsymbol{z}}\left(\pi_{i}^{(n)}\right)=Q\left(\Phi_{\left(R z_{1}, \ldots, R z_{n}\right)}\left(\pi_{i}^{(n)}\right)\right)=Q\left(R z_{i}\right)=z_{i} \quad(i \in\{1, \ldots, n\}) .
$$


To verify that $\Phi_{\boldsymbol{z}}$ satisfies (1.4), let $m \in \mathbb{N}, f_{1}, \ldots, f_{m} \in H_{n}$ and $g \in H_{m}$, and set $k_{i}=\Phi_{\left(R z_{1}, \ldots, R z_{n}\right)}\left(f_{i}\right) \in C(K)$ for $i \in\{1, \ldots, m\}$. Then, on the one hand, (3.7) implies that

$$
\Phi_{\left(\Phi_{z}\left(f_{1}\right), \ldots, \Phi_{\boldsymbol{z}}\left(f_{m}\right)\right)}(g)=Q\left(\Phi_{\left(R Q k_{1}, \ldots, R Q k_{m}\right)}(g)\right),
$$

while on the other,

$$
\Phi_{\boldsymbol{z}}\left(g \circ\left(f_{1} \times \cdots \times f_{m}\right)\right)=Q\left(\Phi_{\left(k_{1}, \ldots, k_{m}\right)}(g)\right)
$$

by (3.7) and (1.4). Since $R Q k_{i}-k_{i} \in \operatorname{ker} Q=J$ for each $i \in\{1, \ldots, m\}$, we can find a neighbourhood $U$ of $s_{0}$ such that

$$
R Q k_{i}(u)=k_{i}(u) \quad(u \in U, i \in\{1, \ldots, m\}) .
$$

Hence, using (3.6) twice, we obtain

$$
\begin{aligned}
\left(\Phi_{\left(R Q k_{1}, \ldots, R Q k_{m}\right)}(g)\right)(u) & =g\left(R Q k_{1}(u), \ldots, R Q k_{m}(u)\right) \\
& =g\left(k_{1}(u), \ldots, k_{m}(u)\right)=\left(\Phi_{\left(k_{1}, \ldots, k_{m}\right)}(g)\right)(u) \quad(u \in U),
\end{aligned}
$$

so that $\Phi_{\left(R Q k_{1}, \ldots, R Q k_{m}\right)}(g)-\Phi_{\left(k_{1}, \ldots, k_{m}\right)}(g) \in J$. Combining this with (3.8)-(3.9), we conclude that (1.4) holds.

Remark 3.2. $\quad$ (i) The reader who prefers a more concrete example may simply consider $K=[0,1]$ and $s_{0}=0$ in Proposition 3.1.

(ii) The hypothesis that $s_{0}$ is the accumulation point of a countably infinite subset of $K$ is necessary, as the example $K=\left[0, \omega_{1}\right]$ (the set of ordinals no greater than the first uncountable ordinal $\omega_{1}$, endowed with the order topology) and $s_{0}=\omega_{1}$ shows: it is a standard fact that the set $X$ given by (3.1) is equal to $C(K)$ in this case.

Our final example involves the following notion. For a locally compact Hausdorff space $\Omega$,

$$
C_{0}(\Omega)=\{f \in C(\Omega): \text { the set }\{t \in \Omega:|f(t)| \geqslant \varepsilon\} \text { is compact for each } \varepsilon>0\}
$$

is a Banach lattice with respect to the pointwise defined operations and the supremum norm $\|\cdot\|_{\infty}$. The evaluation map at a point $t_{0} \in \Omega$ is denoted by $\varepsilon_{t_{0}}$, that is,

$$
\varepsilon_{t_{0}}: f \mapsto f\left(t_{0}\right), \quad C_{0}(\Omega) \rightarrow \mathbb{R} .
$$

We require the following result, which is probably well-known. However, as we have been unable to find it in the literature, we include a proof, which is similar to that of $[1$, Theorem 2.33].

Lemma 3.3. Let $\Omega$ be a locally compact Hausdorff space. A map $\varphi: C_{0}(\Omega) \rightarrow \mathbb{R}$ is a vector lattice homomorphism if and only if $\varphi=c \varepsilon_{t_{0}}$ for some $c \in[0, \infty)$ and $t_{0} \in \Omega$. 
Proof. Each map of the form $c \varepsilon_{t_{0}}$, where $c \in[0, \infty)$ and $t_{0} \in \Omega$, is clearly a vector lattice homomorphism.

Conversely, suppose that $\varphi: C_{0}(\Omega) \rightarrow \mathbb{R}$ is a vector lattice homomorphism, and denote by $C_{c}(\Omega)$ the norm-dense subspace of $C_{0}(\Omega)$ consisting of all compactly supported functions. The restriction of $\varphi$ to $C_{c}(\Omega)$ is a positive linear functional, so the Riesz-Markov Theorem (see for instance [7, p. 352]) implies that

$$
\varphi(f)=\int f \mathrm{~d} \mu \quad\left(f \in C_{c}(\Omega)\right)
$$

for some inner regular Borel measure $\mu$ on $\Omega$. Let $\operatorname{supp} \mu$ be the support of $\mu$, defined as in [7, Exercise 24, pp. 351-352]. If $\operatorname{supp} \mu=\emptyset$, then $\varphi=0$, so we can take $c=0$ and any point $t_{0} \in \Omega$. Otherwise the result will follow provided that we can show that $\operatorname{supp} \mu$ consists of a single point. Assume the contrary, so that supp $\mu$ contains two distinct points, say $t_{1}$ and $t_{2}$. Since $\Omega$ is locally compact and Hausdorff, there are disjoint open, relatively compact subsets $U_{1}$ and $U_{2}$ of $\Omega$ such that $t_{i} \in U_{i}$ for $i \in\{1,2\}$. By Urysohn's Lemma, we can find continuous functions $f_{1}, f_{2}: \Omega \rightarrow[0,1]$ such that $f_{i}\left(t_{i}\right)=1$ and $f_{i}$ vanishes on $\Omega \backslash U_{i}$ for $i \in\{1,2\}$. Note that $f_{1}, f_{2} \in C_{c}(\Omega)$ because $U_{1}$ and $U_{2}$ are relatively compact. Hence, on the one hand, we have

$$
\varphi\left(f_{1}\right) \wedge \varphi\left(f_{2}\right)=\int f_{1} \mathrm{~d} \mu \wedge \int f_{2} \mathrm{~d} \mu>0
$$

because $t_{1}, t_{2} \in \operatorname{supp} \mu$. On the other, $f_{1} \wedge f_{2}=0$ implies that $\varphi\left(f_{1}\right) \wedge \varphi\left(f_{2}\right)=\varphi\left(f_{1} \wedge f_{2}\right)=0$, which is clearly absurd.

Example 3.4. Endow the vector space $X=\mathbb{R}^{2}$ with the lexicographic order:

$$
\left(s_{1}, s_{2}\right) \leqslant\left(t_{1}, t_{2}\right) \Longleftrightarrow\left(s_{1}<t_{1}\right) \text { or }\left(s_{1}=t_{1} \text { and } s_{2} \leqslant t_{2}\right) \quad\left(s_{1}, s_{2}, t_{1}, t_{2} \in \mathbb{R}\right),
$$

and set

$$
x_{1}=(1,0) \in X^{+}, \quad x_{2}=(0,1) \in X^{+} \quad \text { and } \quad \boldsymbol{x}=\left(x_{1}, x_{2}\right) \in X^{2} .
$$

Then $X$ is a vector lattice which is not Archimedean because $n x_{2} \leqslant x_{1}$ for each $n \in \mathbb{N}$.

We claim that $X$ does not admit any positively homogeneous continuous function calculus. More precisely, we shall show that for the particular choice of $\boldsymbol{x}$ given by (3.11), no vector lattice homomorphism $\Phi_{\boldsymbol{x}}: H_{2} \rightarrow X$ with $\Phi_{\boldsymbol{x}}\left(\pi_{i}^{(2)}\right)=x_{i}$ for $i \in\{1,2\}$ exists.

Assume the contrary, and set $\varphi_{i}=\pi_{i}^{(2)} \circ \Phi_{\boldsymbol{x}}: H_{2} \rightarrow \mathbb{R}$ for $i \in\{1,2\}$. The definition (3.10) implies that the first coordinate projection $\pi_{1}^{(2)}: X \rightarrow \mathbb{R}$ is a vector lattice homomorphism, and consequently $\varphi_{1}$ is a vector lattice homomorphism. Recalling the identification (2.2) 
of $H_{2}$ with $C\left(S_{\ell_{\infty}^{2}}\right)$ and using Lemma 3.3, we deduce that $\varphi_{1}=c_{1} \varepsilon_{s}$ for some $c_{1} \in[0, \infty)$ and $s \in S_{\ell_{\infty}^{2}}$. Equation (2.3) in the case $n=2$ implies that

$$
\begin{aligned}
c_{1} & =\varphi_{1}(\mathbf{1})=\pi_{1}^{(2)} \circ \Phi_{\boldsymbol{x}}\left(\left|\pi_{1}^{(2)}\right| \vee\left|\pi_{2}^{(2)}\right|\right) \\
& =\pi_{1}^{(2)}\left(\left|\Phi_{\boldsymbol{x}}\left(\pi_{1}^{(2)}\right)\right| \vee\left|\Phi_{\boldsymbol{x}}\left(\pi_{2}^{(2)}\right)\right|\right)=\pi_{1}^{(2)}\left(\left|x_{1}\right| \vee\left|x_{2}\right|\right)=\pi_{1}^{(2)}\left(x_{1}\right)=1
\end{aligned}
$$

Now the calculation

$$
\pi_{i}^{(2)}(s)=\varphi_{1}\left(\pi_{i}^{(2)}\right)=\pi_{1}^{(2)}\left(\Phi_{\boldsymbol{x}}\left(\pi_{i}^{(2)}\right)\right)=\pi_{1}^{(2)}\left(x_{i}\right)=\delta_{i, 1} \quad(i \in\{1,2\})
$$

shows that $s=(1,0)=x_{1}$, and consequently $\varphi_{1}=\varepsilon_{x_{1}}$.

Although $\pi_{2}^{(2)}$ is not a vector lattice homomorphism, the restriction of $\varphi_{2}$ to $\operatorname{ker} \varphi_{1}$ is a vector lattice homomorphism because

$$
\varphi_{2}(f \vee g)=\pi_{2}^{(2)}\left(\Phi_{\boldsymbol{x}}(f) \vee \Phi_{\boldsymbol{x}}(g)\right)=\pi_{2}^{(2)}\left(\left(0, \varphi_{2}(f)\right) \vee\left(0, \varphi_{2}(g)\right)\right)=\varphi_{2}(f) \vee \varphi_{2}(g)
$$

for every $f, g \in \operatorname{ker} \varphi_{1}$. Since we can identify $\operatorname{ker} \varphi_{1}=\left\{f \in C\left(S_{\ell_{\infty}^{2}}\right): f\left(x_{1}\right)=0\right\}$ with $C_{0}\left(S_{\ell_{\infty}^{2}} \backslash\left\{x_{1}\right\}\right)$, Lemma 3.3 implies that $\varphi_{2} \uparrow_{\operatorname{ker} \varphi_{1}}=c_{2} \varepsilon_{t}$ for some $c_{2} \in[0, \infty)$ and $t=$ $\left(t_{1}, t_{2}\right) \in S_{\ell_{\infty}^{2}} \backslash\left\{x_{1}\right\}$. We have $\pi_{2}^{(2)} \in \operatorname{ker} \varphi_{1}$ because $\pi_{1}^{(2)}\left(x_{2}\right)=0$, and therefore

$$
c_{2} t_{2}=\varphi_{2}\left(\pi_{2}^{(2)}\right)=\pi_{2}^{(2)}\left(\Phi_{\boldsymbol{x}}\left(\pi_{2}^{(2)}\right)\right)=\pi_{2}^{(2)}\left(x_{2}\right)=1,
$$

so that $c_{2}, t_{2}>0$. Take $\lambda \in\left(t_{1} / t_{2}, \infty\right)$ and define $f=\pi_{1}^{(2)} \vee\left(\lambda \pi_{2}^{(2)}\right)-\pi_{1}^{(2)} \in C\left(S_{\ell_{\infty}^{2}}\right)$. Then on the one hand we have

$$
\varphi_{2}(f)=\pi_{2}^{(2)}\left(\Phi_{\boldsymbol{x}}(f)\right)=\pi_{2}^{(2)}\left(x_{1} \vee\left(\lambda x_{2}\right)-x_{1}\right)=0,
$$

while on the other $f \in \operatorname{ker} \varphi_{1}$ because $f\left(x_{1}\right)=0$, and hence

$$
\varphi_{2}(f)=c_{2} f(t)=c_{2}\left(t_{1} \vee\left(\lambda t_{2}\right)-t_{1}\right)=c_{2}\left(\lambda t_{2}-t_{1}\right)>0 .
$$

This is clearly absurd, and this contradiction completes the proof of our claim that $X$ does not admit a positively homogeneous continuous function calculus.

Acknowledgements. We are grateful to Professor Gerard Buskes (Mississippi) for discussing a preliminary version of this work with us.

\section{REFERENCES}

[1] C. D. Aliprantis and O. Burkinshaw, Positive operators, Springer, Dordrecht, 2006, Reprint of the 1985 original.

[2] G. Buskes, B. de Pagter and A. van Rooij, Functional calculus on Riesz spaces, Indag. Math. (N.S.) 2 (1991), 423-436.

[3] G. Buskes and C. Schwanke, Functional completions of Archimedean vector lattices, Algebra Univers. 76 (2016) 53-69. 
[4] J. L. Krivine, Théorèmes de factorisation dans les espaces réticulés, Séminaire Maurey-Schwartz 1973-1974: Espaces L $L^{p}$, applications radonifiantes et géométrie des espaces de Banach, Exp. 22-23, Centre de Math., École Polytech., Paris, 1974.

[5] J. Lindenstrauss and L. Tzafriri, Classical Banach spaces. II. Function spaces, Ergebnisse der Mathematik und ihrer Grenzgebiete 97, Springer-Verlag, Berlin-New York, 1979.

[6] B. de Pagter and A. W. Wickstead, Free and projective Banach lattices, Proc. Royal Soc. Edinb. 145A (2015), 105-143.

[7] H. L. Royden, Real analysis, third ed., Macmillan Publishing Company, New York, 1988.

[8] A. J. Yudin, Solutions de deux problèmes de la théorie des espaces semi-ordonnés, Doklady Akad. Nauk SSSR 23 (1939), 418-422.

Department of Mathematics and Statistics, Fylde College, Lancaster University, LanCASTER, LA1 4YF, United Kingdom

E-mail address: n.laustsen@lancaster.ac.uk

Department of Mathematical and Statistical Sciences, University of Alberta, Edmonton, Alberta T6G 2G1, Canada

E-mail address: troitsky@ualberta.ca 